\title{
Corela
}

Cognition, représentation, langage

HS-34 | 2021

Les noms sous-spécifiés en français: du lexique au discours

\section{Cette chose étrange que ... cette chose en site cataphorique : de certaines spécificités d'un nom atypique}

\section{Céline Benninger}

\section{(Q) OpenEdition}

\section{Journals}

Édition électronique

URL : https://journals.openedition.org/corela/13739

DOI : $10.4000 /$ corela.13739

ISSN : 1638-573X

Éditeur

Cercle linguistique du Centre et de l'Ouest - CerLICO

Référence électronique

Céline Benninger, "Cette chose étrange que ... cette chose en site cataphorique : de certaines spécificités d'un nom atypique », Corela [En ligne], HS-34 | 2021, mis en ligne le 09 décembre 2021, consulté le 13 décembre 2021. URL : http://journals.openedition.org/corela/13739 ; DOI : https:// doi.org/10.4000/corela.13739

Ce document a été généré automatiquement le 13 décembre 2021.

\section{(c) (i) (3) (2)}

Corela - cognition, représentation, langage est mis à disposition selon les termes de la licence Creative Commons Attribution - Pas d'Utilisation Commerciale - Partage dans les Mêmes Conditions 4.0 International. 


\title{
Cette chose étrange que ... cette chose en site cataphorique : de certaines spécificités d'un nom atypique
}

\author{
Céline Benninger
}

\section{Introduction}

1 Le nom chose appartient à ces catégories de noms atypiques que sont les noms sousspécifiés, les noms généraux ou encore les noms dits sommitaux. Nous aurions pu parler aussi de shell nouns, signalling nouns et / ou de carrier nouns ${ }^{1}$. Le nom chose figure d'ailleurs dans les listes signalées par les chercheurs à l'origine de chacune de ces catégories conceptuelles, même si ces listes ne coïncident pas par ailleurs. Notre propos n'est pas de choisir précisément à laquelle de ces catégories le nom chose appartient, d'autant que les frontières et les critères identificatoires de ces dernières - c'est admis -, ne sont pas encore clairement posés. Ce qui nous intéresse davantage, c'est d'aller plus avant dans les arcanes de l'implication de chose dans la cohésion textuelle.

2 Nous avions abordé cette question en étudiant les emplois indéfinis du nom chose dans des séquences bipartites ${ }^{2}$, comme celles des exemples (1) et (2):

(1) Une chose est sûre : l'été sera long à venir.

(2) Je pense à une chose. Quand il fera beau, il faudra veiller à tailler les haies.

Dans ces constructions, chose est le pivot autour duquel s'installent deux séquences, interdépendantes aux niveaux syntaxique, sémantique et pragmatique, et aussi prosodique et intonatif ${ }^{3}$ pour l'oral. Leur interprétation repose sur des processus cataphoriques concomitants à une hypostatisation ou réification. En somme, ces configurations initient et achèvent tout à la fois la création d'un objet de pensée qui serait, respectivement pour les exemples ci-dessus un été long à venir, des haies à tailler quand il fera beau ${ }^{4}$. Ce sont là autant d'entités éphémères, de type ad hoc, dont l'existence 
est conditionnée par son investissement dans la cohésion textuelle. Nous aimerions poursuivre l'exploration des emplois cataphoriques du nom chose, mais, cette fois, lorsqu'il est pris dans les griffes du déterminant démonstratif, comme dans les exemples (3) et (4) :

(3) [...] et cette " tante Bléas ", conscience morale de la famille, avait à cœur de bien traiter le beau-frère. Il avait droit à cette chose rare, un verre de vin. Dans cette nombreuse famille, on faisait à chaque naissance la folie d'acheter un litre de vin. (Ozouf M., Composition française : retour sur une enfance bretonne, 2009)

(4) Dominique, mon mystérieux petit avenir, mon mystérieux « moi », mon petit garçon ou ma petite fille, comment seras-tu, qui seras-tu, est-ce que je saurai faire cette chose la plus difficile de toutes, t'élever ? (Torrès T., Une Française libre : journal 1939-1945, 2000)

Après avoir rappelé les spécificités sémantico-référentielles du nom chose ainsi que celles des sites cataphoriques dans lesquels son emploi indéfini se trouve imbriqué, nous verrons ce qu'il en est de ses emplois démonstratifs.

Deux raisons au moins motivent cette entreprise. D'abord, elle est inédite : même si à

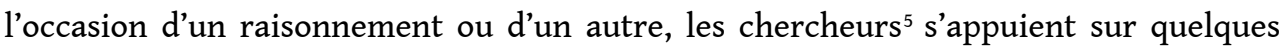
exemples d'emplois démonstratifs des noms atypiques, ces derniers ne sont jamais étudiés per se. Ensuite, le site que nous nous proposons d'étudier sous-tend un certain paradoxe. En effet, il réunit deux éléments dont les fonctions sémantiques et pragmatiques sont en quelque sorte antagonistes: d'une part, le déterminant démonstratif, connu pour construire sa référence en s'appuyant fortement sur le coet/ou le contexte (cf. en particulier les notions d'emplois situationnels gestuels, non gestuels, indirects et les emplois endophoriques référentiels, fidèles, infidèles ou résomptifs ${ }^{6}$ ) tout en recherchant un référent qui se distingue au sein de sa catégorie; d'autre part, le $\mathrm{N}$ chose qui pour sa part et par défaut renvoie à de l'inédit, de l'inconnu et/ou du non encore nommé 7 . En d'autres termes, le SN cette chose met en rapport deux unités linguistiques dont le travail est a priori contradictoire. On peut donc supposer que de tels emplois seront contraints par un certain nombre de paramètres, l'interaction entre le déterminant démonstratif et le nom chose générant des calculs sémantiques importants. Quelles sont ces contraintes? Peut-on tabler sur celles qui commandent à des sites démonstratifs canoniques? A nous de les mettre à jour, de trouver comment le déterminant démonstratif influe sur les règles d'insertion $\mathrm{du} \mathrm{N}$ chose dans le discours, et inversement.

Une dernière chose pour en finir avec les remarques introductives: nous ne proposerons pas d'analyse quantitative de notre corpus. Les sites à l'étude, extraits du corpus de textes postérieurs à 2000 que propose Frantext, et Factiva, ne s'y prêtent pas. Ils présentent en tout cas une telle diversité de réalisations syntaxiques qu'il nous a semblé impossible de les rassembler à des fins statistiques. Une petite information chiffrée toutefois pour donner un ordre de grandeur de la portée du phénomène étudié : sur l'ensemble des occurrences du nom chose (tout type de configurations confondues), le SN cette chose ne représente que $2 \%$ des emplois, soit cinq fois moins que ses emplois indéfinis. Les constructions qui nous intéressent représentent donc un faible nombre de cas au regard des occurrences de chose. 


\section{Rappels}

\subsection{Le nom chose}

7 Quelle que soit l'étiquette attribuée au nom chose, il est admis que son contenu sémantique est loin d'être standard en ce qu'il présente les deux caractéristiques suivantes :

(i) une très grande pauvreté sémantique. Martin, dans le $\mathrm{DMF}^{8}$, définit chose comme désignant une "idée d'intension nulle, c'est-à-dire d'extension maximale, applicable à tout, l'extension étant déterminée par le contexte ; [...]. »

(ii) le corollaire de cette pauvreté sémantique en fait, d'une manière quelque peu paradoxale, un désignateur d'une puissance peu commune puisque, par la voie d'une hypostatisation, il est apte à ériger en individu issu d'une classe quelque chose qui vient à l'existence dans le moment-même de l'énonciation. Ce faisant, il s'installe dans le discours comme un désignateur / une dénomination pour des entités non encore nommées?

8 Ces caractéristiques sont valables pour les exemples (1) et (2). Elles sont aussi particulièrement bien mises en évidence dans des sites comme (5):

(5) Même si tu pédales vite jusqu'à chez toi, tu n'auras pas le temps d'installer le buffet, les invités vont t'attendre à la porte. Je te propose une chose : dis-moi ce dont tu as besoin, donne-moi une liste avec tout ce que tu veux et je vais faire les courses avant. (Mréjen V., Eau sauvage, 2004)

où une chose renvoie à ce que désigne la succession des propositions subséquentes au double point, soit un ensemble d'entités du deuxième ordre ${ }^{10}$, et plus précisément un référent doté d'une dynamicité certaine. Conceptuellement, ce dernier ne correspond pas à une image comme celle d'un objet concret comptable, comme p. ex. vélo, mais plutôt à un objet de pensée animé.

\subsection{Les emplois cataphoriques du SN indéfini une chose ${ }^{11}$}

La nature même de l'indéfini fait que les emplois cataphoriques du SN une chose sont principalement des sites bipartites, comme en (1), (2) ou (5). Dans ce cadre, une chose présente plusieurs caractéristiques :

(i) les rares compléments admis dans le SN une chose restent de ceux qui distinguent un objet au sein d'une classe, d'une série, sans chercher à le décrire. Il s'agit d'adjectifs comme seule, première, dernière, etc. c'est-à-dire de ces adjectifs qui, selon Schnedecker (2002), sont d'un troisième type. De fait, nous retiendrons la quasi-absence de complémentation comme une partie intégrante de l'identité de la configuration du site d'occurrence du SN une chose ${ }^{12}$

(ii) que le SN indéfini soit sujet d'une phrase attributive ou verbale, qu'il soit objet d'un verbe, les énoncés produits relèvent, sur le plan sémantique, de la subjectivité (cf. p. ex. les attributs sûr, certain, clair, acquis, etc.; les verbes comme frapper, étonner, tracasser, chagriner, navrer, donner du contentement à, décider qq'un à, survenir, mettre la puce à l'oreille de

(iii) la subjectivité en question est associée à une première personne (cf. une chose me frappe, je suis frappé par une chose, etc.). Quelle que soit la position syntaxique de une chose dans la première séquence, il pose le locuteur comme le sujet de la pensée exprimée, l'entité psychique qui transcende un ressenti, une opinion, etc.

(iv) S2, en tant qu'antécédent cataphorique, se doit de saturer référentiellement le SN une chose, en respectant l'instruction « S2 apporte un nouveau savoir », voire « un savoir inconnu » directement issue de la subjectivité du locuteur 
(v) la construction hérite de cette organisation une force pragmatique, essentielle pour l'interlocuteur qui se trouve guidé dans le passage vers une connaissance ou une conceptualisation inédite ${ }^{13}$

(vi) le bon fonctionnement du site dépend de la conjonction de ces contraintes.

11 Qu'en est-il des sites du syntagme démonstratif cette chose?

\section{Le site à l'étude : le SN cette chose}

12 L'examen du corpus a fait ressortir deux cas de figure, ayant en commun d'être des exophores mémorielles ou des cas de deixis in absentia ${ }^{14}$. L'un et l'autre partagent bien sûr un certain nombre de caractéristiques avec les configurations typiques de l'emploi indéfini de chose. Via le déterminant démonstratif, les sites à l'étude présentent toutefois des spécificités.

\subsection{Cette chose : démonstratif cataphorique spécifique}

\subsubsection{Description du site}

Le premier type de configuration correspond aux cas identifiés dans la littérature sur la question comme des démonstratifs cataphoriques spécifiques (désormais DCS). Connus pour être des emplois non dociles du démonstratif, les DCS échappent aux emplois classiquement décrits pour les démonstratifs. En effet, comme le dit Kleiber (2004a), ils ne correspondent ni à des emplois déictiques, ni à des emplois situationnels (qu'ils soient gestuels ou non), ni à des emplois anaphoriques puisqu'aucune mention du référent visé n'est extractible du contexte antérieur. Les DCS se distinguent en règle générale par les trois caractéristiques suivantes :

(i) ils dépendent, pour fonctionner, de la présence, à droite de chose, d'une proposition subordonnée, où cette dernière est obligatoire

(ii) ils dénotent, comme leur nom l'indique, un référent particulier, la lecture générique leur étant fermée ${ }^{15}$

(iii) ils confèrent au référent dénoté par le $\mathrm{SN}$ démonstratif un statut informationnel que caractérise la notoriété, en ce sens qu'il fait partie des connaissances encyclopédiques enregistrées dans la mémoire longue des locuteurs.

L'ensemble de ces caractéristiques se trouvent illustrées par des exemples comme (6) et (7) :

(6) Tout le temps qu'on s'emparait des cambrioleurs pour les emmener au poste, la vieille femme resta de marbre, sur son visage le masque de la vertu outragée. Maintenant, comme sonné par la sortie de sa grand-mère, Didier se taisait, accablé par l'énormité de cette chose impensable qu'il sortait entre deux gendarmes, menottes aux poignets, indifférent au sort de son comparse qui continuait de sangloter. (Garat A.-M., Dans la main du diable, 2006)

(7) [...] malgré toutes les barrières et les silences et son inaltérable raideur et certainement la culpabilité elle avait cherché et était parvenue à me dire cette chose qu'obscurément et pour ainsi dire à son insu elle avait eu l'intention de me révéler dès l'instant où elle avait décroché son téléphone pour m'inviter à cette fatidique soirée et, en définitive, ce n'était qu'au tout dernier moment qu'elle avait trouvé la force et la possibilité de desserrer l'étau et de me tendre la main [...]. (Bouillier G., L'Invité mystère, 2004)

Notons que l'on peut trouver aussi, en lieu et place de la proposition subordonnée, des compléments prépositionnels centrés sur un infinitif, comme en (8) : 
(8) Dix fois j'ai failli dire que l'homme dont vous parliez avec tant de ferveur, c'était pour lui que j'étais là... Pour lui que je faisais cette chose insensée d'être chez vous. Mais vous étiez sombre, et froid. Je n'en ai pas eu le courage. (Garat A.-M., Dans la main du diable, 2006)

On constate par ailleurs que dans les exemples (6) et (8), chose est accompagné aussi d'un syntagme adjectival, respectivement impensable et insensée.

Quel que soit le mode du verbe de l'expansion à droite de chose, sa suppression est impossible et compromet l'emploi du démonstratif. La cause en est que les expansions de type adjectival de ces exemples ne parviennent pas, à eux seuls, à satisfaire l'exigence de chose à trouver un référent :

(6)a *Maintenant, comme sonné par la sortie de sa grand-mère, Didier se taisait, accablé par l'énormité de cette chose impensable.

(8)a *Pour lui que je faisais cette chose insensée.

La suppression des adjectifs épithètes semble, par contre, possible et, dépourvus de ces derniers, les exemples (6) et (8) rejoignent alors la configuration de l'exemple (7) :

(6)b Maintenant, comme sonné par la sortie de sa grand-mère, Didier se taisait, accablé par l'énormité de cette chose qu'il sortait entre deux gendarmes, menottes aux poignets, indifférent au sort de son comparse qui continuait de sangloter.

(8)b Pour lui que je faisais cette chose d'être chez vous.

Comment expliquer cet état de fait? Pourquoi les expansions de type verbe sont-elles obligatoires au contraire de celles de type adjectif? En tant que syntagme démonstratif, cette chose joue le rôle de désignateur en ce qu'il doit permettre l'identification d'un référent ${ }^{16}$ et seul un contenu de type verbe semble à même de pouvoir réaliser cela. Comme le montre Riegel (1993: 7), les adjectifs sont des concepts descriptifs ${ }^{17}$ « dont les occurrences particulières (les cas de "rouge(ur) ») ne se réalisent que si elles sont " supportées " par quelque chose qu'elles caractérisent et dont elles présupposent l'existence ». Plus particulièrement, lorsqu'il est épithète, comme c'est le cas dans les DCS, l'adjectif qualificatif se contente d'intégrer, sur le mode déterminatif ou descriptif, une propriété dans les expressions référentielles dont il est syntaxiquement et sémantiquement dépendant. Mais sa capacité s'arrête là : il n'est pas à même de déterminer une classe de référents ${ }^{18}$.

C'est pour cette même raison que l'on peut affirmer que l'adjectif épithète est facultatif.

Bien sûr, du fait même de sa capacité descriptive, l'adjectif peut satisfaire l'exigence imposée par le déterminant démonstratif de mener à un référent qui se distingue au sein de la classe des référents à laquelle il appartient. Via l'adjectif qualificatif peut donc se trouver confortée l'exigence de nouveauté et / ou de distinctivité sur le plan informationnel. Mais l'adjectif ne peut jouer ce rôle qu'à partir du moment où l'identification d'un référent est assurée, ce que seul le complément de type verbe peut faire. En effet, de par leur valeur prédicative, les expansions propositionnelles permettent l'accès au référent visé. En tous cas, les prédications de ces tournures, à travers leur pouvoir classificatoire ou catégorisateur, offrent au syntagme démonstratif la possibilité de satisfaire l'exigence qui lui est propre, celle de correspondre à un référent connu autrement que par sa seule appartenance à la classe des N. Chose, encore plus fortement que n'importe quel $\mathrm{N}$ standard dans une telle configuration, en tant que nom sémantiquement sous-déterminé, ne peut faire autrement que de s'appuyer sur le contenu prédicatif des éléments qu'il installe dans son immédiate dépendance, sa propre architecture sémantique en étant incapable. En définitive, les compléments 
prédicatifs mènent à l'identification du type de référent, les compléments adjectivaux assoient l'opposition du référent visé aux autres référents du même type.

21 Poursuivons l'exploration de ce type de configuration en décrivant plus précisément encore les prédications véhiculées par les compléments que chose installe autour de lui. Il faut en effet revenir sur le statut informationnel des expansions prédicatives du syntagme démonstratif, ces expansions qui précisément mènent à l'identification du référent.

\subsubsection{Les spécificités des SNDém}

Selon Kleiber (2004a \& b), le contenu prédicatif des DCS présente les caractéristiques suivantes :

- il constitue un savoir supposé partagé par le locuteur et l'interlocuteur

24 - il doit, pour répondre aux exigences du déterminant démonstratif, puiser dans le stock des connaissances inscrites dans la mémoire longue, connaissances communes et partagées. Dans de tels sites, «le démonstratif donne l'information apportée dans la mémoire discursive comme une information ancienne, déjà enregistrée, parce que ce n'est qu'à cette condition que l'appariement référentiel qui le caractérise trouve matière à s'appliquer.» (2004b : 242)

Cela peut poser un problème avec chose dans la mesure où la référence associée à ce $\mathrm{N}$ n'a pas d'existence présupposée et stable et que, par voie de conséquence, il est impossible de puiser dans un quelconque stock de connaissances antérieures le concernant. Examinons alors de plus près les propositions gravitant autour de chose et pour ce faire, reprenons les exemples (6) et (8):

(6)c cette chose impensable qu'il sortait entre deux gendarmes, menottes aux

poignets, indifférent au sort de son comparse qui continuait de sangloter

(8)c cette chose insensée d'être chez vous

Lorsque le $\mathrm{N}$ d'un syntagme du type DCS est chose, les connaissances apportées par le contenu propositionnel ne peuvent se prévaloir d'aucune autre caractéristique que celle d'être en parfaite adéquation avec le discours dans lequel il s'insère (cf. la matching constraint). On peut le constater à partir des exemples : elles appartiennent aux connaissances inférables à partir du contexte et sont en parfaite adéquation avec ce dernier. Plus largement, bien sûr, les connaissances nécessaires à l'identification du référent visé par cette chose seront aussi en adéquation avec nos connaissances encyclopédiques du monde.

\subsubsection{Bilan}

Pour ce qui est des DCS lorsque le $\mathrm{N}$ est chose, trois caractéristiques seront donc à retenir :

3) le nom chose doit s'entourer de contenus prédicatifs

4) ces derniers ont pour fonction de déterminer le référent visé, sachant que ce dernier est inédit

5) ils le font sur la base de connaissances accessibles à partir du contexte d'emploi.

Les DCS en chose se distinguent donc d'autres DCS sur le plan de la notoriété qu'il convient d'accorder aux informations prédicatives. Elles ne sont pas exclusivement issues « des connaissances communes déjà partagées » (Kleiber, 2004a : 242). On l'a vu, la notoriété des connaissances issues des prédications est ici directement liée au 
contexte d'emploi, à la situation d'énonciation. Ce n'est qu'à cette condition que le référent de chose peut émerger pour s'installer dans la mémoire immédiate ou dans le contexte discursif. Ce faisant, la contrainte d'appariement du déterminant démonstratif se trouve amplement satisfaite. Ce site exige donc un travail de type inférentiel sur le plan sémantico-pragmatique. Et même si la configuration et les contraintes imposées sont différentes de ses emplois indéfinis, le site d'occurrence étudié est une nouvelle fois le lieu d'une hypostatisation fortement soutenue par des dispositifs syntaxiques et sémantico-pragmatiques.

\subsection{Cette chose : « véritable » site cataphorique}

29 Le deuxième type de configuration cataphorique du SN cette chose se distingue des précédents en ce que le processus diaphorique sur lequel il repose relève véritablement de la cohésion discursive. Les DCS sont ce que Halliday \& Hasan (1976 : 68) appellent des cataphores structurelles, soit des cataphores qui sont «simply a realization of a grammatical relationship within the nominal group and he has no cohesive, textforming function ». Les sites dont il est question ci-dessous sont, eux, des cataphores textuelles et, en tant que telles, sont de véritables vecteurs de cohésion discursive. En effet, comme nous le verrons :

30 - l'interprétation du syntagme en chose dépend de l'interprétation d'un autre segment textuel ${ }^{19}$, quelquefois segmental, quelquefois plus large (cf. la notion de cataphore résomptive)

31 - ce segment se verra recruté à la droite de chose et hors du syntagme démonstratif

32 - entre ces deux segments existe une relation de dépendance de type sémanticoréférentielle

33 - l'interprétation de l'une étant fonction de l'autre, ce type de configuration participe à la cohésion textuelle.

34 Pour préciser ces points et certains autres encore, prenons les exemples (9) et (10) :

(9) C'était la première fois de ma vie que je rencontrais quelqu'un de mon âge avec qui je pouvais partager cette chose en commun : avoir eu des parents très militants. (Linhart V., Le jour où mon père s'est tu, 2008)

(10) Je lui ai dit, tu sais je suis sûre qu'il y a plein de projets passionnants en Europe aussi... Nous faisions le même métier... Il a hoché la tête et m'a répondu cette chose

qui m'a longtemps hantée : Tu ne penses qu'à toi. (Gavalda A., La Consolante, 2008)

Dans ces exemples et comme prévu, le $\mathrm{SN}$ en chose trouve à se saturer référentiellement en cherchant dans son contexte droit un segment idoine, soit respectivement avoir eu des parents très militants et tu ne penses qu'à toi. Il ne semble peser sur l'antécédent aucun contrainte syntaxique particulière ${ }^{20}$. Les réalisations de l'antécédent sont des syntagmes nominaux (SN pour (3) et supra (11)), des propositions nominales (cf. supra (15)), des propositions infinitives ((9) et supra (16)), des propositions au sens d'unité phrastique dont le noyau verbal est conjugué ((10) et supra les ex. (14) et (17)), des prises de parole par le passage au style direct (supra (12)), des suites de phrases ou de propositions, éventuellement coordonnées (supra (13)). 


\subsubsection{Avec et sans DCS}

\section{Quelquefois la catap
comme $(11)-(13)^{21}$ :}

(11) Prendre l'air nous fera du bien. Peut-être qu'en roulant le long de la rivière s'apaisera cette chose que je sens entre nous depuis hier ou plus longtemps, ce silence aux marques imperceptibles et pour moi immédiatement reconnaissables. (Cusset C., New York, journal d'un cycle, 2009)

(12) [...] je remercie l'ange qui m'a écarté les mâchoires contaminées par sa pudeur pour formuler, à cet instant, cette chose qui va tellement de soi qu'il paraissait superflu d'y mettre des mots, des mots de la tribu, sans même prendre le temps de leur donner un sens plus pur: "Vous ne le savez peut-être pas, ai-je dit, je n'ai jamais aimé que vous. » (Billetdoux M., Un peu de désir sinon je meurs, 2006)

(13) Arrivés au portail de la maison, une dernière scène, superbe, je le sens, pour la réalisation de cette chose-là qu'à défaut d'autres mots on appelle l'amour : il laisse la radio (Yves Duteil, "Le petit pont de bois ») et je le caresse avec la bouche, jusqu'à sa jouissance, là, dans la voiture arrêtée allée des Lozères. (Ernaux A., Se perdre, 2001)

La coexistence des deux types de cataphores n'a rien de systématique. Le nombre d'exemples relevés sans DCS est loin d'être anecdotique. On peut le constater avec les exemples (14) à (17) ${ }^{22}$ :

(14) Dans cette langueur de l'aveu qui venait à ses lèvres ensommeillées, c'était à Endre qu'elle disait cette chose vraie, amoureuse et si triste : c'est une belle langue... (Garat A.-M., Dans la main du diable, 2006)

(15) [...] elle se remit debout avant de revenir vraiment à elle, les oreilles pleines du tintamarre qui ne cessait pas, tandis qu'elle voyait, très précisément, cette chose extraordinaire : Terrier tassé sur la banquette, tenant son bras ensanglanté. (Garat A.-M., Dans la main du diable, 2006)

(16) À des milliers de kilomètres, quelque part sur les routes encombrées par les fuyards, ma mère roule dans la vieille De Dion de ma grand-mère, emmenant avec elle son père et sa mère, et ses deux enfants âgés d'un an et de trois mois. C'est sans doute à ce moment-là que mon père tente cette chose folle, traverser le désert pour s'embarquer en Algérie à destination du sud de la France afin de sauver sa femme et ses enfants et les ramener avec lui en Afrique. (Le Clézio J.-M. G., L'Africain, 2004)

(17) Parfois, ce rêve revient plusieurs nuits de suite. Tantôt je suis à Paris, tantôt chez Annie, tantôt à Chanteloup, ou à Lectoure, et toujours il y a cette chose horrible : un fossé est survenu entre le passé et mon retour. (Torrès T. Une Française libre: journal 1939-1945, 2000)

Trois choses sont à souligner :

- du point de vue des expansions autour de chose, on a soit un SADJ, soit une expansion sous la forme d'une proposition subordonnée. Contrairement aux DCS, les cas de cumuls entre des expansions de type adjectif et de type verbe ne sont pas exemplifiés dans notre corpus

- ce dernier n'a, par ailleurs, révélé aucune occurrence du syntagme cette chose nu, alors que c'était largement le cas pour les emplois indéfinis cataphoriques de chose. Cet état de fait est probablement lié à l'instruction issue du déterminant démonstratif qui oblige à recruter un référent distinct, au sein de sa catégorie, des autres référents. Or, nous l'avons vu, l'adjectif est le type d'expansion apte à accomplir une telle tâche. Cette configuration n'est pourtant pas exclue. On va le voir dans le point suivant 
41 - le fonctionnement des DCS est moins contraint qu'il ne l'est dans les cataphores structurelles. Les expansions de type verbe semblent en effet facultatives. On peut le vérifier sur (12), par exemple :

(12)a je remercie l'ange qui m'a écarté les mâchoires contaminées par sa pudeur pour formuler, à cet instant, cette chose : «Vous ne le savez peut-être pas, ai-je dit, je n'ai jamais aimé que vous. »

42 La suppression des compléments prédicatifs n'est pas irrémédiablement fatale, comme cela l'est pour les DCS. Lorsque l'antécédent recouvre une prise de parole, le site semble pouvoir se passer des expansions. Nous retiendrons toutefois que les syntagmes nus ne sont pas complètement satisfaisants, pour la raison que nous avons évoqué au point précédent: un site démonstratif est soumis à une exigence de nouveauté et/ou de distinctivité dans le recrutement de son référent. La disparition des expansions, quelles qu'elles soient, affaiblit les chances du site de répondre favorablement à une telle exigence.

43 En somme, d'une manière tout à fait logique, la coexistence, dans un même site, des deux types de cataphores centrées sur le même $\mathrm{N}$ allège la nécessité du SN en chose à trouver le référent visé. Ce travail se partage entre les deux cataphores, l'une et l'autre ayant ce même objectif. Et s'il y avait une hiérarchie à établir entre les cataphores, c'est évidemment en faveur de la cataphore textuelle que pencherait la balance. C'est elle qui semble essentielle pour le site.

\subsubsection{Sites démonstratifs vs sites indéfinis}

Les cataphores textuelles démonstratives valident grosso modo les propriétés des sites cataphoriques du SN indéfini (cf. \$1.2.). En résumé, à l'instar des sites indéfinis, les cataphores textuelles démonstratives de chose :

- se jouent entre deux séquences textuelles, séparées par une pause, quelle qu'elle soit, tout en étant liées par une relation de dépendance

- recrutent un antécédent qui apporte une information de type "savoir nouveau »

- héritent de cette configuration d'une force pragmatique, essentielle pour l'interlocuteur qui se trouve accompagné dans la saisie d'une connaissance ou une conceptualisation inédite et reposant sur la subjectivité d'une instance pensante.

Les sites démonstratifs se distinguent toutefois des sites indéfinis sur un point: les SADJ des sites démonstratifs disposent d'une plus grande latitude quant à leur type sémantique. Les syntagmes démonstratifs des exemples sans DCS (cf. entre autres, les exemples (14) à (17) : cette chose vraie / extraordinaire / folle / horrible) ne sont pas exclusivement des adjectifs de troisième type qui, du reste, n'apparaissent dans aucun exemple du corpus alors qu'ils étaient pléthore dans les sites indéfinis. Cette différence peut s'expliquer par le fait qu'avec le démonstratif, chose aspire à renvoyer à un élément distinct d'autres éléments du même type alors qu'avec l'indéfini, l'urgence est d'accéder au statut d'élément ou d'objet de pensée. Et adjectifs qualificatifs et adjectifs du troisième type sont respectivement et parfaitement adaptés à ces tâches.

Enfin, une dernière remarque pour compléter la description des sites de cataphores démonstratives textuelles : la substitution de l'indéfini au démonstratif n'est pas exclue d'emblée. Nous pouvons le vérifier avec les exemples investis dans cette étude. Cet état de fait est intéressant et mérite en tout cas qu'on lui trouve une explication, ce que nous essayons de faire maintenant en revenant à la notion de mode de donation d'un 
référent. Selon Theissen (1997: 109), la façon pour un syntagme indéfini de présenter un référent repose sur une opération qui permet d'une part "l'extraction d'une occurrence à partir de la classe préconstruite des $\mathrm{N}$ et d'autre part et simultanément, un ancrage spatio-temporel de cette occurrence par le prédicat». L'existence du référent associé à un $\mathrm{SN}$ indéfini est donc fonction des circonstances propres au discours auquel il appartient et l'article indéfini suppose un mode de donation référentiel particulier qui équivaut à la présentation dans le discours d'un référent donné comme inédit. Du côté du démonstratif, toujours selon Theissen (1997: 414), outre la contrainte d'appariement, l'emploi du déterminant démonstratif invite les locuteurs à focaliser, via une procédure d'isolement, leur attention sur le référent visé, ce dernier devant être trouvé dans le contexte d'énonciation avec, d'une part, une contrainte de type nouveauté informationnelle et, d'autre part, une fonction classifiante pour un référent appréhendé comme une entité non nommée, non encore classée. Cette propriété est d'autant plus fortement marquée dans les cataphores démonstratives de chose que ces dernières relèvent de la deixis am Phantasma, ou démonstratif de point de vue. En effet, selon Kleiber (2003:38), le pointage de ce type de deixis «s'effectue dans des espaces ou domaines mentaux. Elle fonctionne avec l'aide de la "vue et de l'oreille mentales» (geistigen Auges und Ohres) rendant possible un déplacement «dans le domaine du remémorable absent et même dans le domaine de l'imagination » (ins Reich des abwesend Erinnerbaren oder gar ins Reich der Phantasie) (Bühler, 1934 : 124)».

En somme, les modes de donation, qu'ils soient indéfinis ou démonstratifs, présentent tous deux une exigence de nouveauté. Peut-on de fait considérer la possibilité de remplacer dans les sites à l'étude le déterminant démonstratif par le déterminant indéfini comme découlant du vague sémantique inhérent à chose ? Le référent de chose n'étant jamais connu par avance, quel que soit le site dans lequel il travaille, la caractéristique «être nouveau, innommé, inédit » ne peut être que satisfaite. Le rôle des déterminants est donc moins décisif et, d'une certaine manière, il n'est laissé à leur charge que la façon dont la nouveauté est installée. Ils n'en portent pas l'entière responsabilité.

\section{En guise de conclusion : une synthèse}

51 Nous voudrions dans cette dernière section mettre en perspective l'ensemble des données réunies pour et par cette étude, ainsi que celles de travaux antérieurs.

Ont été étudiés pour chose, différents sites d'occurrences qui sont soit :

- des configurations syntagmatiques ${ }^{23}$ :

- le syntagme peu de chose, original pour le singulier du N, alors qu'il est comptable

- les syntagmes du type "Les choses de + N abstrait déterminé » comme les choses du monde / de l'amour / de la vie / de la religion, etc.)

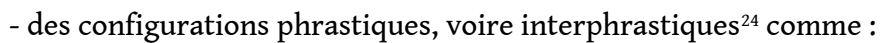

- les sites de cataphores indéfinis

- les sites de cataphores démonstratifs

- les constructions bipartites de type pseudo-clivées. 

immanquablement un nombre certain de contraintes qui lui reviennent en propre. L'emploi de toute unité linguistique obéit à des règles, de quelque niveau qu'elle soit. Mais chose va au-delà et son bon fonctionnement en langue suppose pour ainsi dire des règles typiques de chose d'abord, de chacun de ses types d'emploi ensuite. Les variables sont immanquablement de deux types: le contenu sémantique de chose, d'une part, le recours à des adjoints structuraux d'autre part. De plus, il semble qu'entre les deux, les choses s'équilibrent selon un principe de vase communicant. Plus le contenu sémantique de chose est faible, ou potentiellement large ou ouvert, plus le nombre, le rôle et les modalités des adjoints structuraux est important. On l'a vu ici, avec les sites cataphoriques démonstratifs où, pour pallier sa faible détermination sémantique, chose fait appel à de nombreux soutiens d'ordre syntaxique et sémantique, dans et autour de son syntagme d'occurrence. Si, à l'inverse, le contenu sémantique de chose est verrouillé, parce qu'il désigne, par exemple, le sexe ou une entité marquée [+h], le soutien syntaxique périphérique s'en trouve significativement allégé. On peut le constater à partir d'exemples comme (18) ou (19) :

(18) [...] : non seulement les femmes sont nues sous leurs vêtements, mais elles ont toutes cette chose miraculeuse entre les jambes, et le plus troublant c'est qu'elles l'ont tout le temps, même quand elles n'y pensent pas. (Carrère E., Un roman russe, 2007)

(19) Ecoutez mon bonheur, Gabrielle : c'est qu'en tombant, grâce à cette chute... cette nuit, j'ai perdu cette chose, enfin... l'enfant est parti. (Garat A.-M., Dans la main du diable, 2006)

où, en définitive, la seule codification nécessaire à la saisie du référent visé est celle du déterminant. On retrouve cette même réalité dans tous les emplois de chose, semble-t-il. Même dans les emplois les plus anodins, il ne fait jamais comme les autres. On achète, trouve, repeint un vélo, même un truc, mais on achète, trouve, repeint quelque chose plutôt que une chose. Au minimum, chose demande le soutien d'un déterminant autre que celui auquel on aurait recours avec un nom standard. Un peu comme s'il était inhérent à ce nom d'envoyer un message d'alerte.

De fait, on peut se poser la question suivante : un nom général n'est-il pas un nom qui dispose tout simplement de la langue pour réunir ce qu'il lui faut pour atteindre un référent en devenir? Il a pour ce faire toute latitude dans le sens où il peut mobiliser autant d'éléments qu'il faut pour y arriver. Quelquefois, le choix du bon déterminant suffit (sachant qu'il n'est pas celui qu'aurait demandé un nom standard). D'autres fois, il va encapsuler du contenu dans des éléments fournis par le cotexte. D'autres fois encore, comme dans les DCS, il s'équipe de compléments prédicatifs dont on peut, d'une certaine manière, considérer qu'ils sont comme l'expression de ses traits sémantiques définitoires. Quoiqu'il en soit, son implication dans la cohésion textuelle est une partie intégrante de son identité.

Corela, HS-34 | 2021 


\section{BIBLIOGRAPHIE}

Adler, S. (2014a). « Evaluation, référence et noms généraux attitudinaux ». Langue Française 184/4, 93-108.

Adler, S. (2014b). « L'événement fortuit à travers le prisme du 'nom général' ». Neophilologica 26, 217-231.

Adler, S. (2017). « Les noms généraux - « shell nouns » - participent-ils à une lecture taxinomique de type Hiérarchie-être? ». Syntaxe et Sémantique 18 / 1, 45-66.

Adler, S. \& Eshkol-Taravella, I. (2016). « Noms généraux et complexité discursive ». In O.

Galatanu, A.-M. Cozma \& A. Bellachhab (éds), Représentations du sens linguistique : les interfaces de la complexité. Bruxelles : Peter Lang, 51-64.

Adler, S. \& Moline, E. (éds) (2018). Les Noms généraux. Langue Française 198.

Benninger, C. (2014). « La question de la définition sémantique du nom atypique chose ». Travaux de Linguistique 69, 35-55.

Benninger, C. (2015a). « Petite grammaire de peu de chose ». L'information Grammaticale 145, 41-49.

Benninger, C. (2015b). « Les choses de + N abstrait déterminé : étude syntactico-sémantique ». In E. Buchi, J.-P. Chauveau \& J.-M. Pierrel (éds), Actes du XXVIIe Congrès international de linguistique et de philologie romanes (Nancy, 15-20 juillet 2013), 3 volumes. Strasbourg : Société de linguistique romane / ÉLiPhi, 36-48.

Benninger, C. (2017). « Une chose [X] : P » : une conjonction de contraintes ». In C. Lachet, L. Meneses-Lerín \& A. Roig (éds), Contraintes linguistiques. A propos de la complémentation nominale, Gramm-R 38. Bruxelles : Peter Lang, 77-95.

Benninger, C. (2018a). « Considérons une chose: P. Construction syntactico-sémantique entre consécutivité et simultanéité ». Dixit Grammatica, Paris : L’Harmattan, 73-91.

Benninger, C. (2018b). « La seule chose que je sais, c'est que je ne sais rien : le N chose et les pseudoclivées. Scolia 32, 69-94.

Bühler, K. (1934). “Sprachtheorie. Die Darstellungsfunktion der Sprache”[Das Organon-Modell]. Verlag von Gustav Fischer : Iéna 1934. Théorie du langage, Agone, 2009.

Frantext https://www.frantext.fr/

Flowerdew, J. (2003). "Signalling Nouns in discourse”. English for Specific Purposes 22, 329-346.

Flowerdew, J. \& Forest, R. (2015). "Signalling Nouns in English: A Corpus-based Discourse Approach". Cambridge: Cambridge University Press.

Francis, G. (1994). "Labelling discourse: an aspect of nominal-group lexical cohesion”. In M. Coulthard (ed.), Advances in written text analysis. London: Routledge, 83-101.

Gerhard-Krait, F. \& Vassiliadou, H. (2014). « Lectures taxinomique et / ou floue appliquées aux noms : quelques réflexions... ». Travaux de Linguistique 69, 57-75.

Gerhard-Krait, F. \& Vassiliadou, H. (2017). « Lectures taxinomique, approximative et floue : quelques pistes supplémentaires, Présentation ». Syntaxe et Sémantique 18, 11-18.

Groupe de Fribourg (2012). Grammaire de la période. Berne : Peter Lang.

Halliday, M. A. K. \& Hasan, R. (ed.) (1976). Cohesion in English. London, New York: Longman. 
Hawkins, J. (1978). Definiteness and indefiniteness. A Study in reference and grammaticality prediction. London: Croom Helm.

Ivanic, R. (1991). “Nouns in search of a context”. International Review of Applied Linguistics XXIX(2), 93-114.

Kleiber, G. (1987). « Mais à quoi sert donc le mot chose? Une situation paradoxale ». Langue Française 73, 109-128.

Kleiber, G. (2003). « Adjectifs démonstratifs et point de vue ». Cahiers de Praxématique 41, 33-54.

Kleiber, G. (2004a). « Sémantique, référence et discours : le cas des démonstratifs cataphoriques spécifiques ». In A. Auchlin et al. (éds), Structures et discours. Mélanges offerts à Eddy Roulet. Québec : Editions Nota bene, 231-245.

Kleiber, G. (2004b). « Anticipation, mémoire et démonstratifs cataphoriques ». In R. Sock \& B. Vaxelaire (éds), L'anticipation à l'horizon du Présent. Sprimont : Pierre Mardaga Editeur, 221-236.

Kleiber, G. (2005). « Des démonstratifs bien énigmatiques : les démonstratifs cataphoriques génériques ». In C. Dobrovie-Sorin (éd.), Noms nus et généricité. Saint-Denis : Presses Universitaires de Vincennes, 65-95.

Kleiber, G. (2007). « Des démonstratifs mémoriels aux démonstratifs de point de vue ». In L. Begioni \& C. Muller (éds), Problèmes de sémantique et de syntaxe. Hommage à André Rousseau. Villeneuve d'Ascq : Editions du Conseil Scientifique de l'Université Charles de Gaulle-Lille 3, 373-392.

Legallois, D. (2008). « Sur quelques caractéristiques des noms sous-spécifiés ». Scolia 23, 109-127. Legallois, D. \& Grea, P. (2006). « L'objectif de cet article est de... Construction spécificationnelle et grammaire phraséologique ». Cahiers de Praxématique 46, 161-186.

Lyons, J. (1980). Sémantique linguistique. Paris : Larousse.

Mahlberg, M. (2005). “English general Nouns; a corpus theoretical Approach”. AmsterdamPhiladelphia: John Benjamins.

Riegel, M. (1993). «Grammaire et référence : à propos du statut sémantique de l'adjectif qualificatif ». L'Information Grammaticale 58, 5-10.

Schmid, H.-J. (2000). "English abstract Nouns as conceptual Shells”. Berlin, New-York: Mouton de Gruyter.

Schmid, H.-J. (2007). "Non-compositionality and emergent Meaning of lexico-grammatical Chunks: a corpus Study of Noun Phrases with sentential Complements as Constructions". Zeitschrift für Anglistik und Amerikanistik 55.3, 313-340.

Schnedecker, C. (éd.) (2002). L'adjectif sans qualité(s), Langue Française 136.

Theissen, A. (1997). Le choix du nom en discours. Paris-Genève : Droz.

Wiederspiel, B. (1995). « Sur quelques aspects de la saisie démonstrative ». Pratiques :

linguistique, littérature, didactique 85, 113-125.

Winter, E. O. (1977). "A clause relational approach to English texts: a study of some predictive lexical items in written discourse”. Instructional Science 6, 1-92. 


\section{NOTES}

1. Sur la définition et l'exploitation de ces concepts, cf. en particulier Adler (2014a \& b), Adler \& Eshkol-Taravella (2016), Adler \& Moline (2018), Flowerdew (2003), Flowerdew \& Forest (2015), Francis (1994), Halliday \& Hasan (1976), Ivanic (1991), Legallois (2008), Legallois \& Gréa (2006), Mahlberg (2005), Schmid (2000, 2007), Winter (1977).

2. Cf. Benninger (2017, 2018a).

3. Pour ces deux derniers traits, cf. la Grammaire de la Période (2012) du Groupe de Fribourg.

4. Cf. la note 2, les travaux cités explicitant ce point.

5. Cf. p. ex. Adler (2017), Mahlberg (2005), Schmid (2000), etc.

6. Cf. pour une synthèse sur ce point, p. ex. Kleiber (2007).

7. Cf. Kleiber (1987) et Benninger (2014, 2017 et 2018a).

8. http://www.atilf.fr/dmf/definition/chose. Notons que cet article ne fait aucune mention de la dichotomie du massif/comptable. Cf. aussi Benninger (2014).

9. Cf. note 7 .

10. Cf. Lyons (1980: 77 sqq).

11. Les rappels faits ici sont issus de Benninger (2017 \& 2018a).

12. Le constat est le même pour les sites définis de chose, dans une position pseudo-clivée (cf. Benninger 2018b).

13. Plus généralement, on peut dire que le $\mathrm{SN}$ en chose bénéficie d'une mise en relief (cf. en particulier ses emplois proches des constructions pseudo-clivées, Benninger 2018b).

14. Cf. Wiederspiel (1995).

15. Il existe des démonstratifs cataphoriques génériques (DCG, Kleiber 2005 \& 2007) qu'illustre l'exemple phare: J'aime ces longs cigares italiens que fume Clint Eastwood dans les westerns. Dans la mesure où le syntagme qui nous intéresse est au singulier, l'accès à de telles lectures lui semble $a$ priori impossible.

16. Cf. la notion de matching constraint de Hawkins (1978) traduite par Kleiber (2004b) par contrainte d'appariement et selon laquelle le déterminant démonstratif fait correspondre de manière explicite le référent à un objet identifiable dans le contexte situationnel ou textuel.

17. Par opposition aux concepts typants qui, eux, présentent une autonomie référentielle (Riegel, $1993: 7)$

18. Cf. aussi Kleiber (2004b : 240).

19. Cf. Halliday \& Hasan $(1976: 5,8,11$, etc.).

20. Nous avions fait le même constat pour les emplois indéfinis et cataphoriques de chose (cf. Benninger, 2018a, §2.2.2).

21. Cf. également l'exemple (10), infra.

22. Cf. également les exemples (3) et (9), infra.

23. Cf. Benninger (2015 a \& b).

24. Cf. Benninger $(2017,2018 a \& b)$.

\section{RÉSUMÉS}

Le nom atypique chose intègre, en tant que noyau de syntagme démonstratif, la place de pivot autour duquel s'installent deux séquences, interdépendantes aux niveaux syntaxique, sémantique et pragmatique. Sur le plan sémantico-référentiel, l'interprétation de telles 
configurations repose sur des processus cataphoriques concomitants à une hypostatisation. A quels types de contraintes est soumis un tel site? En répondant à cette question, nous montrerons que le $\mathrm{N}$ chose, pour pallier sa pauvreté sémantique, nécessite que soient respectées, dans son proche environnement, un certain nombre de paramètres d'ordre sémanticopragmatique et syntaxique. Ce faisant, il agit comme un véritable facteur de cohésion textuelle.

The atypical noun chose integrates, as a demonstrative phrase, the pivot place around which two sequences, interdependent at the syntactic, semantic and pragmatic levels, are installed. At the semantic and referential level, the interpretation of such configurations is based on cataphoric processes associated with hypostatization. To what types of constraints is such a site submitted? By answering this question, we will show that the $\mathrm{N}$ chose, in order to overcome its semantic poverty, requires a certain number of semantic, pragmatic and syntactic parameters to be respected in its close environment. In doing so, he acts as a real factor of textual cohesion.

\section{INDEX}

Keywords : Chose, general nouns, cataphora, demonstratives phrases, hypostatization, semantics, reference

Mots-clés : Chose, noms généraux, noms sous-spécifiés, cataphore, démonstratif, hypostatisation, sémantique, référence

\section{AUTEUR}

\section{CÉLINE BENNINGER}

Université de Strasbourg, EA 1339 LiLPa - Scolia 